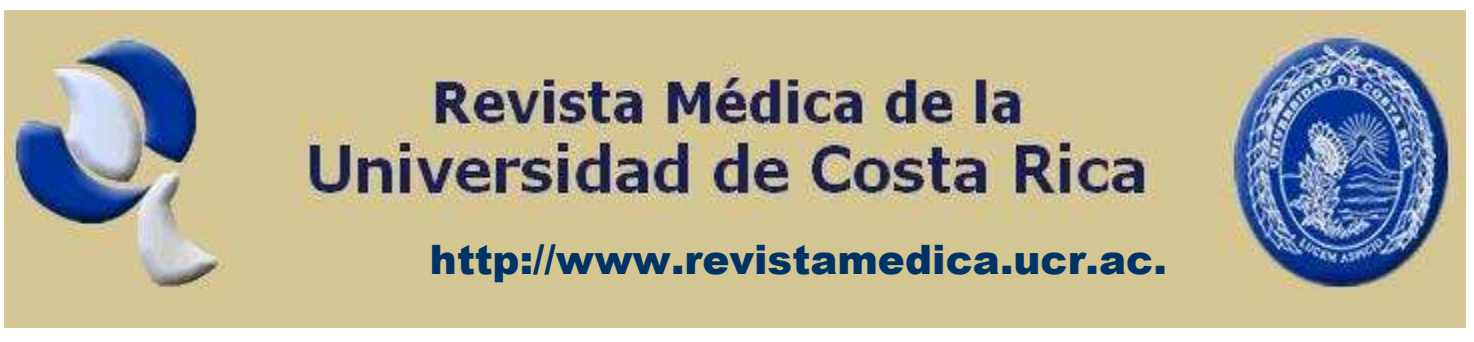

Artículo original

\title{
Uso de Gabapentina en los Servicios de Neurología y Medicina Interna del Hospital México en el 2007
}

\author{
Agüero Navarro, Nuria ${ }^{1}$; Herrera Arias, Cristina ${ }^{2}$ y Aburto Varela, Daniela ${ }^{3}$ \\ 1. Farmacéutica, C.C.S.S, San José, Costa Rica. 2. Profesora, Instituto de Investigaciones \\ Farmacéuticas (INIFAR), Facultad de Farmacia, Universidad de Costa Rica, San Pedro, San José, \\ Costa Rica. 3. Farmacéutica, Servicio de Farmacia, Hospital México, San José, Costa Rica.
}

Resumen: El consumo de gabapentina en el Hospital México ha ido en aumento año tras año desde su primera adquisición en el 2004, por lo que el objetivo del presente estudio es evaluar la prescripción de gabapentina, $300 \mathrm{mg}$, en los servicios de Neurología y Medicina Interna del Hospital México de enero a diciembre del 2007, con base en los criterios establecidos por el Comité Central de Farmacoterapia en la V Acreditación para Comités Locales de Farmacoterapia por medio de la revisión de expedientes clínicos. Se encontró que en el $10 \%(n=9)$ de los pacientes se empleó la gabapentina para tratar la epilepsia $(n=$ 3) o la neuralgia postherpética $(n=6)$, las cuales corresponden a las dos patologías aprobadas en la V Acreditación; sin embargo, solamente en el 33\% (n = 3) de estos casos se llevó a cabo siguiendo la línea de prescripción descrita en el V documento de acreditación. En el 90\% $(n=80)$ de los casos fue empleada en el tratamiento de patologías no aprobadas por la $\mathrm{V}$ Acreditación. Se encontró también que la dosis efectiva más comúnmente utilizada de gabapentina fue de $300 \mathrm{mg}$ tres veces al día. Se concluye que el empleo de gabapentina $300 \mathrm{mg}$ en el Hospital México no se realiza según lo estipulado en la V Acreditación para Comités Locales de Farmacoterapia y por lo tanto el aumento del consumo de la gabapentina en este hospital se debe, en mayor parte, a su empleo en patologías no aprobadas por el Comité Central de Farmacoterapia. 
Palabras clave: gabapentina, Hospital México, dolor neuropático, neuralgia postherpética, epilepsia, medicamentos no LOM.

Recibido: Agosto 2009. Aceptado: Septiembre 2009. Publicado: Septiembre 2009.

Abstract: The consumption of gabapentin in the Hospital Mexico has increased during the years since its first acquisition in 2004. The objective of this study is to evaluate the prescription of $300 \mathrm{mg}$ gabapentin in the Internal Medicine and Neurology Services of the Hospital Mexico from January to December of 2007, according to the criteria established by the Comité Central de Farmacoterapia in the $V$ Acreditación para Comités Locales de Farmacoterapia, by the study of the clinical record. We found that in $10 \%(n=9)$ of the patients, the gabapentin was used to treat epilepsy $(n=3)$ or the postherpetic neuralgia $(n=6)$, which correspond to the approved pathologies in the $V$ Acreditación para Comités Locales de Farmacoterapia; however, only $33 \%(n=3)$ of these cases were treated following the prescription line in agreement with the institutional guidelines. In $90 \%(n=80)$ of the cases the medication was used in the treatment of pathologies unapproved by the institution already mentioned. It also was found that the effective dose commonly used of gabapentin was $300 \mathrm{mg}$, three times per day. We conclude that the use of $300 \mathrm{mg}$ gabapentin in the Hospital México doesn't agree with the guidelines of the $V$ Acreditación para Comités Locales de Farmacoterapia and the increase of the gabapentin consumption in this hospital is due to, in most of the cases, the treatment of pathologies unapproved by the Comité Central de Farmacoterapia.

Keywords: Gabapentin, Hospital México, neuropathic pain, postherpetic neuralgia, epilepsy, no LOM medicines.

\section{Introducción}

La gabapentina fue introducida en el mercado en 1993 y aprobada como medicamento anticonvulsivante por la Food and Drug Administration (FDA) de Estados Unidos para tratar las crisis convulsivas parciales en adultos, con generalización consecutiva o sin ella. Posteriormente en el año 2002 fue aprobada por este organismo para el tratamiento de la neuralgia postherpética. [1, 2] Por otro lado, la European Medicines Agency (EMEA) aprueba el empleo de gabapentina en las crisis convulsivas parciales con o sin generalización secundaria, y en el

tratamiento del dolor neuropático periférico. [1, 3]

Desde su introducción al mercado, su uso se ha extendido ampliamente. Según un estudio realizado por la FDA en el 2002, alrededor de un $95 \%$ de los pacientes que utilizan gabapentina lo hacen para tratar patologías en las cuales su empleo no ha sido aprobado por este organismo regulatorio. [4]

La normativa que rige la utilización de la gabapentina en la Caja Costarricense del Seguro Social es dictada por el Comité Central de 
Farmacoterapia y plasmada en los documentos de Acreditación para Comités Locales de Farmacoterapia. El primer documento que incluye la gabapentina fue el publicado en el 2003 que corresponde a la III Acreditación para Comités Locales de Farmacoterapia (CCF-1898-12-2003) donde se aprobó su empleo en dolor neuropático y epilepsia. [5] Desde ese entonces sus indicaciones aprobadas han variado; actualmente el uso de la gabapentina fue restringido exclusivamente para el tratamiento de la neuralgia post-herpética. [6] A pesar de lo expuesto anteriormente, la investigación se basó en lo estipulado en la $\mathrm{V}$ Acreditación para Comités Locales de Farmacoterapia (CFF-89205-06), que es la que abarca el periodo en estudio, donde el fármaco se encuentra aprobado para su empleo en neuralgia postherpética como cuarta línea de tratamiento tras la utilización de analgésicos no narcóticos, AINES, antidepresivos tricíclicos $\mathrm{y}$ anticonvulsivantes $y$ en epilepsia refractaria luego de haber agotado las alternativas terapéuticas disponibles en la lista oficial de medicamentos (LOM). [7]

Tras la inclusión de la gabapentina como medicamento no LOM, ésta fue adquirida por el Hospital México a partir del 2004. Desde entonces, año tras año, se ha observado un aumento en la compra del medicamento y por tanto en su consumo. $[8,9,10]$ Este aumento en la compra y consumo de gabapentina genera inquietud debido a que no se conoce con certeza si el motivo de este incremento se debe a una mayor prescripción según los lineamientos institucionales, o si por el contrario, las prescripciones del fármaco se realizan bajo diagnósticos no incluidos en la V Acreditación para Comités Locales de Farmacoterapia.

El objetivo del presente estudio fue evaluar la prescripción de gabapentina 300 mg en los servicios de Neurología y Medicina Interna del Hospital México, con base en los criterios establecidos por el Comité Central de Farmacoterapia en la V Acreditación para Comités Locales de Farmacoterapia, durante el 2007.

\section{Métodos}

Esta investigación es un estudio observacional descriptivo sin patrocinio externo. El listado de pacientes a los cuales se les prescribió gabapentina de enero del 2007 a diciembre del 2007 se obtuvo del Sistema Integrado de Farmacias (SIFA). La selección de los pacientes se realizó mediante la aplicación de los siguientes criterios de inclusión: pacientes mayores de 18 años, ambos sexos y exclusión: pacientes con solamente un retiro, pacientes que solo recibieron el medicamento estando hospitalizados y pacientes que no retiraron su medicamento luego de tres meses consecutivos siempre y cuando no fuera por indicación médica.

De la revisión de los expedientes clínicos correspondientes se obtuvieron los siguientes datos para cada paciente: diagnóstico de prescripción, tratamiento, línea de prescripción seguida para lo cual se anotaron los diversos medicamentos empleados en orden cronológico hasta la prescripción de la gabapentina $\mathrm{y}$ dosis efectiva de gabapentina prescrita. 
En los casos en que la gabapentina fuera prescrita para una patología distinta a la neuralgia postherpética o la epilepsia, no se realizó la descripción de la línea de prescripción seguida, solamente se anotó el diagnóstico para el cual fue indicada.

En el análisis de datos se determinó si la gabapentina fue empleada correctamente según los lineamientos institucionales. Se consideró un empleo correcto de la gabapentina en los casos en que fue prescrita para las patologías aprobadas y siguiendo la línea de prescripción según la $\mathrm{V}$ Acreditación para Comités Locales de Farmacoterapia. Se hizo empleo de tablas y gráficos que mostraran los distintos resultados obtenidos.

\section{Resultados}

De un total de 212 pacientes extraídos del programa SIFA a los cuales se les prescribió gabapentina durante el 2007 se excluyeron 68 pacientes porque realizaron un solo retiro del medicamento en el año, 33 por abandono del tratamiento y 15 porque solo recibieron el medicamento estando hospitalizados. Se excluyeron 7 pacientes más, a pesar de que cumplían los criterios de inclusión/exclusión porque el expediente no estaba disponible o por ausencia de datos en el mismo. Por lo tanto, se seleccionaron finalmente 89 pacientes.

Tras la revisión de los expedientes clínicos se obtuvo que en un $10 \%$ (n =
9) de los pacientes se empleó gabapentina para tratar las dos patologías aprobadas en la V Acreditación para Comités Locales de Farmacoterapia, donde el 33\% ( $n=3$ ) corresponde al uso de gabapentina en pacientes con epilepsia refractaria y el $66 \%(\mathrm{n}=6)$ a pacientes con neuralgia posherpética; sin embargo, sólo el 33\% $(\mathrm{n}=3)$ de los casos en que se empleó gabapentina para tratar la epilepsia refractaria o la neuralgia posherpética se llevó a cabo siguiendo la línea de prescripción descrita en la $\mathrm{V}$ Acreditación para Comités Locales de Farmacoterapia (Tabla 1). Por lo tanto, tomando en cuenta tanto la indicación como la línea de prescripción, sólo el $3 \%(\mathrm{n}=3)$ de la gabapentina prescrita por los Servicios de Medicina Interna y Neurología del Hospital México durante el 2007 se realizó siguiendo los lineamientos institucionales.

El $90 \%(n=80)$ restante de las prescripciones de gabapentina se realizó para tratar patologías no aprobadas por la $\mathrm{V}$ Acreditación para Comités Locales de Farmacoterapia. La Figura 1 expone de manera porcentual la distribución de cada una de ellas, la Tabla 2 muestra un mayor detalle del tipo de patología.

Se encontró también que la dosis efectiva utilizada en un $100 \%$ de los casos de neuralgia postherpética y epilepsia fue de $300 \mathrm{mg}$ tres veces al día. 
Tabla 1. Línea de prescripción seguida por los Servicios de Medicina Interna y Neurología del Hospital México durante el 2007 en los casos en que se indicó gabapentina para las patologías aprobadas en la V Acreditación para Comités Locales de Farmacoterapia.

\begin{tabular}{|c|c|c|c|c|c|}
\hline Paciente & $\begin{array}{c}\text { Tratamiento } \\
\mathbf{\#} \mathbf{1}\end{array}$ & $\begin{array}{c}\text { Tratamiento } \\
\mathbf{\# ~ 2}\end{array}$ & $\begin{array}{c}\text { Tratamiento } \\
\mathbf{\# ~ 3}\end{array}$ & $\begin{array}{c}\text { Tratamiento } \\
\mathbf{\# ~ 4}\end{array}$ & $\begin{array}{c}\text { Uso } \\
\text { correcto }\end{array}$ \\
\hline 1 & PHE/VPA/CBZ & PHE/VPA & VPA & GBP & SI \\
\hline 2 & CLZ & CLZ+VPA & CLZ+CBZ & GBP & SI \\
\hline 3 & VPA & GBP & - & - & NO \\
\hline 1 & GBP & - & - & - & NO \\
\hline 2 & CBZ y CLZ & DCF+VPA+AMT & VPA+CLZ+ACD & GBP & SI \\
\hline 3 & GBP & - & - & - & NO \\
\hline 4 & GBP & - & - & - & NO \\
\hline 5 & CBZ & CBZ+AMT & GBP & - & NO \\
\hline 6 & AMT & GBP & - & - & NO \\
\hline
\end{tabular}

Abreviaturas: PHE fenitoína, VPA ácido valproico, CBZ carbamazepina, GBP gabapentina, CLZ clonazepam, DCF diclofenaco, AMT amitriptilina, ACD Acetaminofén con codeína.

Figura 1. Patologías no avaladas por la V Acreditación para Comités Locales de Farmacoterapia para las cuales se prescribe gabapentina por los Servicios de Medicina Interna y Neurología del Hospital México durante el 2007.

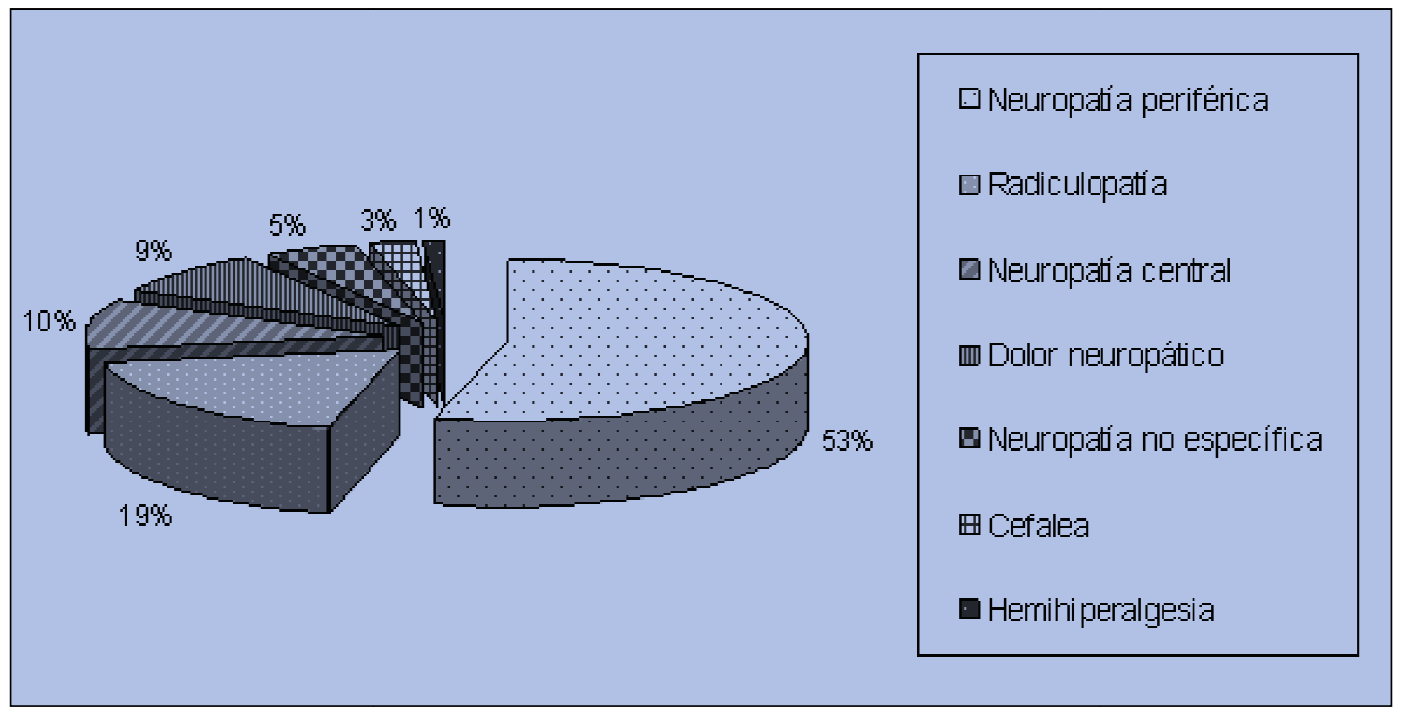


Tabla 2. Detalle de las patologías no avaladas por la V Acreditación para Comités Locales de Farmacoterapia para las cuales se prescribe gabapentina por los Servicios de Medicina Interna y Neurología del Hospital México durante el 2007.

\begin{tabular}{|c|c|}
\hline Patología para la cual se prescribe Gabapentina & Total de pacientes \\
\hline Polineuropatía & 17 \\
Diabetes mellitus & 9 \\
\hline Neuralgia del trigémino & 5 \\
Sensitiva & 3 \\
\hline Sensitivo motora & 2 \\
No específica & 7 \\
\hline Lumbociática & 7 \\
Plexo braquial & 3 \\
\hline Cervicalgia & 2 \\
Compresiva & 1 \\
\hline Diabetes Mellitus & 1 \\
No específica & 1 \\
\hline Síndrome talámico & 3 \\
Mielopatía torácica & 2 \\
\hline Esclerosis múltiple & 2 \\
\hline Post-accidente cerebro vascular & 1 \\
\hline Dolor neuropático & 7 \\
\hline Neuropatía (no específica) & 4 \\
\hline Cefalea & 2 \\
\hline Hemihiperalgesia & 1 \\
\hline & $\mathbf{8 0}$ \\
\hline & TOTAL \\
\hline
\end{tabular}




\section{Discusión}

Al evaluar la prescripción de gabapentina $300 \mathrm{mg}$ en los casos en que fue utilizada para las patologías indicadas en la $\mathrm{V}$ Acreditación, se observa que la línea de prescripción seguida en el tratamiento de la epilepsia incluye en la medicación previa los anticonvulsivantes disponibles en la institución como lo son la fenitoína, ácido valproico, carbamazepina y clonazepam y en dos casos la gabapentina fue empleada como cuarta línea de tratamiento siguiendo los lineamientos institucionales (Tabla 1).

En el único caso en que la gabapentina fue utilizada según la normativa institucional para el manejo de la neuralgia postherpética se puede observar como la línea terapéutica empleada incluye anticonvulsivantes solos 0 en combinación con antidepresivos tricíclicos y analgésicos no esteroideos previo a la utilización de la gabapentina como cuarta línea de tratamiento.

En el resto de pacientes en los que se utilizó la gabapentina para tratar la neuralgia postherpética o la epilepsia, no se encontró documentación acerca de la medicación previa al uso de gabapentina que respaldara su uso. En tres de los casos el fármaco fue utilizado como tratamiento de primera línea y en los otros tres casos no se agotaron las medidas terapéuticas con que cuenta la institución.

Cabe mencionar que el Hospital México por ser un centro de salud clase A donde la gran mayoría de los pacientes son referidos desde los centros de atención primaria de sus comunidades, se carece de la documentación referente a la historia farmacoterapéutica del paciente desde el inicio de su padecimiento. Si bien es cierto se debe realizar una anamnesis completa, en algunas ocasiones no se especificó en el expediente los antecedentes patológicos y terapéuticos suficientes para aclarar el motivo de la prescripción del fármaco en cuestión.

Respecto a la dosis de gabapentina empleada en los pacientes con neuralgia postherpética o epilepsia, como se mencionó, en la totalidad de los casos se prescribió una cápsula de $300 \mathrm{mg}$ de gabapentina tres veces al día. Esta indicación coincide con la descrita en el documento de la V Acreditación para Comités Locales de Farmacoterapia para el empleo de la gabapentina en la neuralgia postherpética e igualmente coincide con la dosis reportada por los entes reguladores internacionales FDA y EMEA. [1]

La utilización de gabapentina para patologías no aprobadas por la $\mathrm{V}$ Acreditación para Comités Locales de Farmacoterapia fue mucho mayor que para las patologías indicadas en este documento, $90 \%$ y $10 \%$ respectivamente. Se observó un predominio del empleo de gabapentina en la neuropatía periférica que representa un $53 \%$ de los casos no aprobados, seguida por las radiculopatías con un $19 \%$ y en tercer lugar se encuentra el dolor neuropático de origen central con un 10\% (Figura 1).

Es importante aclarar que en algunos casos fue imposible obtener más datos con respecto a los diagnósticos durante la revisión de expedientes ya que muchas veces no se disponía de suficiente información, los diagnósticos no eran claros o el padecimiento se encontraba en 
estudio. Por ejemplo, en algunos sólo se indicaba como diagnóstico la presencia de dolor neuropático siendo este un síntoma de diversas etiologías que también es presentado por los demás pacientes.

Al analizar las indicaciones de gabapentina aprobadas en la V Acreditación para Comités Locales de Farmacoterapia y compararlas con las aprobadas por los organismos regulatorios internacionales observamos que, respecto a la FDA, no existe discrepancia alguna ya que las indicaciones aprobadas por esta entidad son las mismas que las que rigen a nivel institucional en el período de estudio. [11, 12] Por otro lado la EMEA tiene aprobado el uso de gabapentina en las convulsiones parciales con o sin generalización secundaria, lo cual coincide con la indicación institucional; sin embargo, en el tratamiento del dolor neuropático este organismo autoriza una utilización mucho más extensa del fármaco al no especificar el tipo de neuropatía periférica para la cual se puede prescribir el medicamento, ya que solamente indica "para el tratamiento del dolor neuropático periférico". [3]

La revisión de la literatura referente a los estudios de eficacia del empleo de gabapentina en las diversas patologías no aprobadas por la FDA, muestra que en la gran mayoría de los casos se carece de estudios randomizados, multicéntricos, doble ciego, controlados que arrojen resultados que demuestren una eficacia estadísticamente significativa. En el caso específico de la neuropatía periférica por diabetes mellitus el único estudio en que se evaluó la eficacia de la gabapentina contra placebo demostró mejoría en un $60 \%$ de los pacientes, pero las dosis empleadas fueron mayores a los 2,4 gramos diarios [2] y las dosis empleadas en los pacientes del estudio en curso fueron de 300-900 mg por día. El uso de gabapentina en la neuralgia del trigémino no ha sido respaldado aún con resultados estadísticamente significativos. [4, 13] Las radiculopatías conforman un grupo de patologías importantes, donde la lumbociática es el subgrupo más representativo y respecto a esta se carece de estudios que respalden el beneficio del empleo de la gabapentina en su tratamiento. [14] En el caso de los dos pacientes con cefalea tratados con gabapentina, uno de ellos con cefalea vascular y otro con cefalea inducida por cirugía craneal, solo existen estudios del empleo de este fármaco como profiláctico de ataques de migraña que demostraron mejoría estadísticamente significativa con dosis diarias de 1,2 gramos [13] y en el caso de este paciente se utilizó una dosis de $900 \mathrm{mg}$ al día. En la neuropatía central se han realizado estudios del empleo de gabapentina en la esclerosis múltiple que han demostrado cierto grado de mejoría pero la cantidad de pacientes es muy pequeña como para reflejar resultados definitivos. [13]

\section{Conclusiones}

El presente estudio muestra que sólo el $3 \%$ de las prescripciones de gabapentina por los Servicios de Medicina Interna y Neurología del Hospital México durante el 2007 se realizaron según lo estipulado en la $\mathrm{V}$ Acreditación para Comités Locales de Farmacoterapia. En el $90 \%$ de los casos se prescribe la gabapentina para patologías no aprobadas en este documento y en el $7 \%$ de los casos no se sigue la línea de prescripción indicada en este documento para las patologías aprobadas, debido a que no se 
agotan las opciones terapéuticas disponibles a nivel institucional antes de considerar su uso.

El aumento de consumo de la gabapentina en el Hospital México en los años posteriores a su inclusión como medicamento no LOM se debe a su empleo en patologías no aprobadas en la $\mathrm{V}$ Acreditación para Comités Locales de Farmacoterapia y no a un aumento en las dosis ya que la dosis efectiva de la gabapentina más utilizada en el Hospital México para el tratamiento tanto de la epilepsia como de la neuralgia postherpética es de $300 \mathrm{mg}$ tres veces al día. Esta tendencia es la observada a nivel mundial.

La utilización de gabapentina en las patologías no aprobadas en la $\mathrm{V}$ Acreditación para Comités Locales de Farmacoterapia tiene escaso soporte desde el punto de vista de la medicina basada en la evidencia.

\section{Recomendaciones}

La dirección del Servicio de Farmacia, con el fin de velar por el uso racional de los medicamentos, debería establecer como pauta la solicitud de la aprobación por el Comité Local de Farmacoterapia para el tratamiento con gabapentina a los pacientes que la requieran. De esta manera se garantizaría que el médico tratante realice la petición formal ante el Comité Local de Farmacoterapia presentando las pruebas que respalden la utilización del fármaco en cada paciente de forma individualizada.

Se debería crear conciencia en la comunidad médica de los servicios de Medicina Interna y Neurología sobre el uso racional de los medicamentos e informar acerca de las patologías para las que está aprobado el uso de gabapentina y las líneas de prescripción según la normativa institucional, reforzando también la práctica de la medicina basada en la evidencia.

Se recomienda que el Comité Central de Farmacoterapia dé seguimiento a los nuevos estudios sobre el uso de gabapentina en patologías no contempladas en el documento de acreditación con el fin de modificar las indicaciones para las cuales se aprueba la utilización del fármaco en cuestión.

\section{Referencias}

1. Lacy Ch, Armstrong L, Goldman M and Lance L. Drug Information Handbook. 15va ed. United States: American Pharmaceutical Association, Lexi Comp. 2006-2007. 826-828 pp.

2. Wiffen PJ, McQuay HJ, Edwards JE and Moore RA. Gabapentina para el dolor agudo y crónico (Revisión Cochrane traducida). En: La Biblioteca Cochrane Plus, 2006; 4. Oxford: Update Software Ltd. Disponible en: http://www.update-software.com.

(Traducida de The Cochrane Library, 2006 Issue 4. Chichester, UK: John Wiley \& Sons, Ltd.).

3. EMEA: European Medicines Agency. Human Medicines-Referrals: List of Referred Applications. Annex I, II, III [Internet]. London (UK); [consultado 2008 Mar 25]. Disponible

en: http://www.emea.europa.eu/htms/human/r eferral/2006.htm.

4. Mack A. Examination of the Evidence for OffLabel Use of Gabapentin. The Journal of Managed Care Pharmacy 2003; 9(6):559568. 
5. Caja Costarricense de Seguro Social, Comité Central de Farmacoterapia. III Acreditación de Comités Locales de Farmacoterapia. Medicamentos no incluidos en la lista oficial para tratamiento agudo o crónico; 2003 Dic. Reporte número: CCF-1898-12-2003). 20 p.

6. Caja Costarricense de Seguro Social, Comité Central de Farmacoterapia. VI Acreditación para Comités Locales de Farmacoterapia. Medicamentos no incluidos en la LOM para tratamiento agudo o crónico; 2008 Ene. Reporte número: CCF-0002-01-08.11 p.

7. Caja Costarricense de Seguro Social, Comité Central de Farmacoterapia. V Acreditación para Comités Locales de Farmacoterapia. Medicamentos no incluidos en la LOM para tratamiento agudo o crónico; 2006 May. Reporte número: CFF-892-05-06.12 p.

8. Dirección de Farmacia Hospital México. Plan Anual Operativo 2008.

9. Bodega Principal de Farmacia Hospital México. Informes anuales de bodega 20042007.
10. Caja Costarricense del Seguro Social, Farmacia Hospital México. Reporte de Consumos Totales de Medicamentos por Servicio: Gabapentina $300 \mathrm{mg}$, periodo 01/01/07 al 31/01/07. 2008 Abr. Reporte número: RptSIFA014.

11. Blengio J, Rivera B, Di Girolamo G. Fármacos eficaces para el tratamiento de las epilepsias. En: Goodman A, Hardman JG, Limbird L. Bases Farmacológicas de la Terapéutica. 10ma ed. McGraw-Hill; 2002. p. 529-547.

12. Gold Standar Inc. Gabapentin [internet]. Tampa (FL); [consultado 2008 Mar 10]. Disponible http://www.clinicalpharmacology.com

13. Herrera J. Gabapentine for the management of other types of neuropathic pain. Revista de la Sociedad Española del Dolor 2002; 9: 3742.

14. Grice GR, Mertens MK. Gabapentin as a potential option for treatment of sciatica [Resumen]. Pharmacotherapy 2008; 28(3): 397-402.

\section{Correspondencia:}

Cristina Herrera Arias,

Instituto de Investigaciones Farmacéuticas (INIFAR), Facultad de Farmacia, Universidad de Costa Rica.

Tel: 2511-4299, fax 2225-3574.

Correo electrónico: cristina.herrera@ucr.ac.cr. 